\title{
Metal Atom Markers for Imaging Epitaxial Molecular Self-Assembly on Graphene by Scanning Transmission Electron Microscopy
}

\author{
Ja Kyung Lee, ${ }^{1}$ Ibrahim Bulut, ${ }^{2}$ Michel Rickhaus, ${ }^{2}$ Yuewen Sheng, ${ }^{1}$ Xiang Li,${ }^{3}$ Grace. G. D. \\ Han, ${ }^{3}$ G. Andrew D. Briggs, ${ }^{1}$ Harry L. Anderson, ${ }^{2}$ Jamie H. Warner ${ }^{* 1}$
}

${ }^{1}$ Department of Materials, University of Oxford, Parks Road, Oxford, OX1 3PH, United Kingdom ${ }^{2}$ Department of Chemistry, University of Oxford, Chemistry Research Laboratory, Oxford, OX1 3TA, United Kingdom

${ }^{3}$ Department of Chemistry, Brandeis University, Waltham, 02453, Massachusetts, USA

*Email: Jamie.warner@materials.ox.ac.uk

\begin{abstract}
Direct imaging of single molecules has to date been primarily achieved using scanning probe microscopy, with limited success using transmission electron microscopy due to electron beam damage and low contrast from the light elements that make up the majority of molecules. Here, we show single complex molecule interactions can be imaged using annular dark field scanning TEM (ADF-STEM) by inserting heavy metal markers of Pt atoms and detecting their positions. Using the high angle ADF-STEM $Z^{1.7}$ contrast, combined with graphene as an electron transparent support, we track the 2D monolayer self-assembly of solution-deposited individual linear porphyrin hexamer (Pt-L6) molecules and reveal preferential alignment along the graphene zigzag direction. The epitaxial interactions between graphene and Pt-L6 drives a reduction in the inter-porphyrin distance to allow perfect commensuration with the graphene. These results
\end{abstract}


demonstrate how single metal atom markers in complex molecules can be used to study large scale packing and chain bending at the single molecule level.

Keywords: ADF-STEM, porphyrins, single molecule, TEM, graphene

Self-assembly of molecules on atomically well-defined surfaces is a promising method for fabrication of the nanometer-sized functional systems. ${ }^{1}$ Conjugated porphyrin oligomers and polymers, either in their linear or cyclic form, have attracted attention in the field of molecular organic electronic devices such as field-effect transistors, solar cells, organic photovoltaics, and single-molecule electronics, ${ }^{2-8}$ because of their unusually fast delocalisation of charge and energy.9-12 ${ }^{9}$ Template-directed synthesis ${ }^{13,14}$ can enable a diverse range of metalloporphyrin oligomer architectures with application in the design of organic semiconductor. Therefore, an understanding of the structure of individual porphyrin oligomer chains and their self-assembly on surfaces at the nanoscale becomes essential for their solid-state integration in devices and surfaces.

Most studies to date of molecular self-assembly on surfaces have been done using scanning probe microscopy (SPM), which has been proven as an ideal technique to image 2D monolayer packing and ordering. ${ }^{15-22}$ However, SPM typically struggles with high through-put screening of molecular self-assembly, making studies difficult and slow when relying on SPM. The other main imaging technique for atomic and nanoscale studies is transmission electron microscopy, but these high energy beams often decompose molecules rapidly under the required dose to obtain sufficient contrast from the light elements that make up the majority of molecular systems. Some success has been made in imaging and characterizing the interactions of small molecules by phase contrast TEM by using very thin electron beam transparent supports, such as the inner region or the surface of carbon nanotubes and graphene. ${ }^{23-28}$ The periodic structure of graphene is also possible to 
remove from images by filtering in reciprocal space to obtain images of molecules as if they are suspended in free space. However, the range of molecules that can be imaged clearly by TEM is generally limited to those that are relatively stable under the electron beam and isolated, because electron beam irradiation can induce intermolecular reactions that change the intrinsic packing behavior, and even transform organic self-assembled monolayer to a $2 \mathrm{D}$ crystalline sheet. ${ }^{29-33} \mathrm{In}$ fullerenes this is observed as fusion together to form nanotubes, ${ }^{34-36}$ or in the case of metal containing molecules this causes the release of metal atoms and the formation of metal nanoclusters and the graphitization of the carbon atoms..$^{26,37}$

The use of annular dark field scanning TEM for molecular imaging has been limited due to the higher damage it induces compared to low-dose phase contrast imaging. Phase contrast imaging at low-dose has been boosted recently with single electron sensitive direct imaging cameras that provide high contrast. ${ }^{38}$ However, ADF-STEM offers contrast that scales as $Z^{1.4-1.7}$ and offers opportunity to discriminate heavier atoms from the carbon backbone in complex molecular systems. ${ }^{39}$ To date, the combination of ADF-STEM with graphene supports has been successful in tracking contrast from heavy Pt metal atoms in azo-benzene photoswitching molecules revealing mechanical trans-cis transitions of individual molecules, 2D packing of $\mathrm{C} 60$ in graphene sandwiches, and Gd-based metallofullerene fusion dynamics on graphene. ${ }^{40-42}$ The ambition to use metal atom markers for ADF-STEM studies of molecules dates back to beyond 30 years ago, with work on using iridium clusters, ${ }^{43}$ but to date there has been little success in observing periodic uniform spacings of single metal atom markers beyond two atoms using ADFSTEM. Expanding this to more complex molecular systems is crucial for its further uptake as a characterization tool. 
The inherent challenge in ADF-STEM imaging is the massive electron dose needed to obtain sufficient contrast from single carbon atoms in order to resolve single molecule structure, and most molecules cannot handle this and approaches for low-dose imaging have been pursued. But relying on imaging carbon atoms requires the substrate support to be pristinely clean, something which is extremely difficult for solution deposited molecules. Here we take a different direction for studying single molecules and their self-assembly on graphene. We focus on using multiple heavy Pt atoms as marker points in a complex molecule and use gentle imaging conditions sufficient to obtain contrast from single Pt atoms, which prevents excessive damage to the molecular monolayers. Linear porphyrin hexamers are synthesized with Pt centers providing six sites of strong contrast in ADF-STEM. This enables the study of 2D monolayer self-assembly on graphene surfaces by ADF-STEM, with the ability to track long range order of stacking. Furthermore, it enables the inter-porphyrin distance to be determined as well and compared to theory, revealing distortions and single molecule bending and structural contractions. This approach can be expanded to other complex molecular systems that can include multiple heavy atoms.

\section{Results and Discussion}

Figure 1a shows a schematic diagram of a platinum $(\mathrm{Pt})$ porphyrin hexamer with termination groups and Figure $1 \mathrm{~b}$ and $1 \mathrm{~d}$ show top and side-views of the atomic structure of a Ptporphyrin linear hexamer (Pt-L6). The four-coordinate Pt atom is in the plane of the porphyrin and the 25 -atom porphyrin core is planar. The meso-aryl substituents are twisted at an angle of $73 \pm 9^{\circ}$ to the mean plane of the porphyrin. ${ }^{44}$ The expected Pt-Pt distance of a butadiyne-linked porphyrin oligomer is $\sim 1.35 \mathrm{~nm}$, according to crystallographic analysis of a zinc porphyrin dimer ${ }^{45}$ and smallangle X-ray scattering of a zinc porphyrin hexamer. ${ }^{12}$ The porphyrin units have long side chains 
to promote solubility in organic solvents (See Supporting Information to find the detailed remarks on organic synthesis). Figure 1c shows an overview of our TEM specimen observed under the scanning electron microscope (SEM) mode at low magnification, where dark circles are suspended graphene and white area is a thick SiN membrane. Deposited porphyrin hexamer molecules on graphene is schematically drawn in Figure 1e.
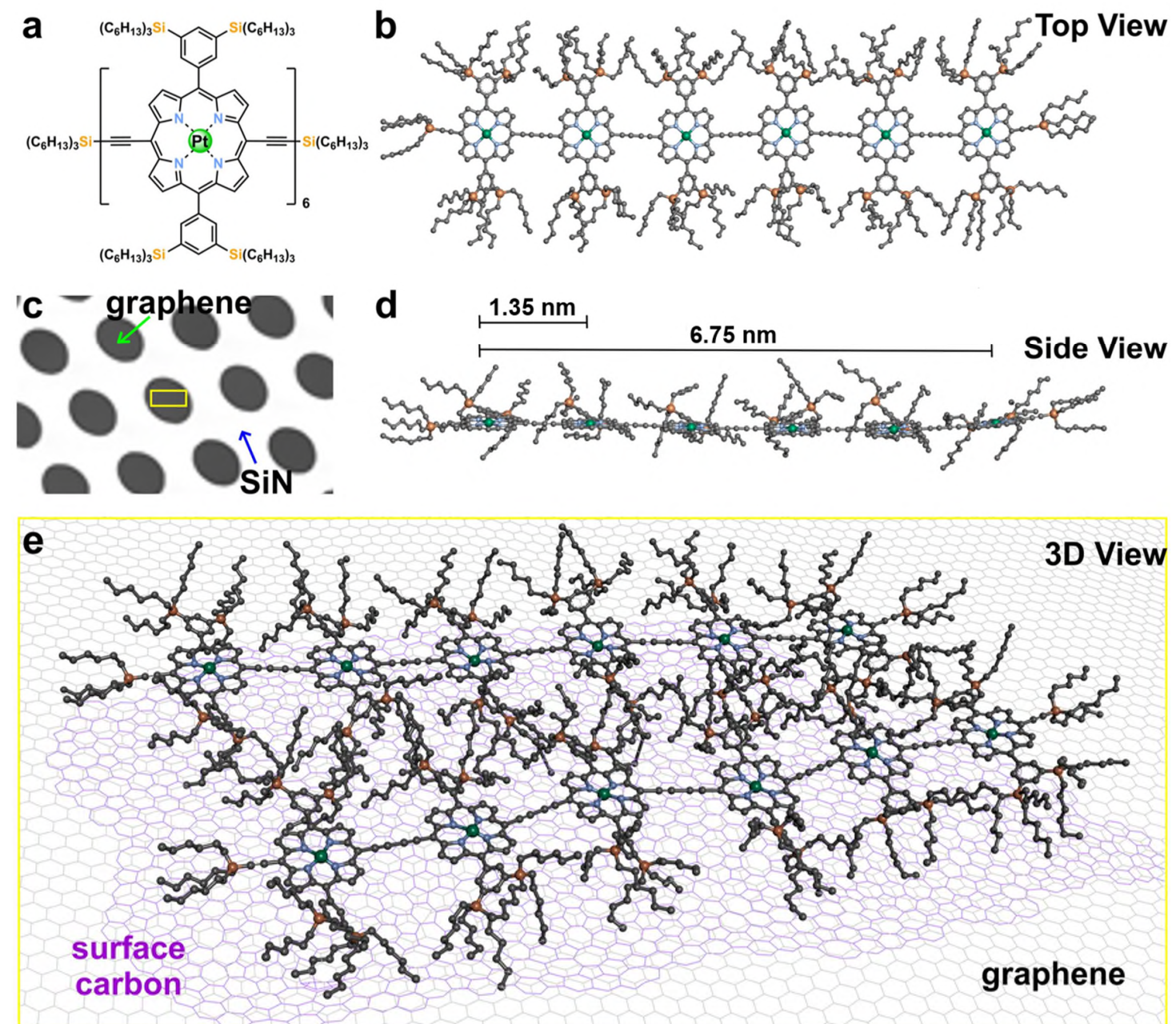

Figure 1. Overview of the study. (a) Schematic of a Pt-porphyrin monomer, where an integer number 6 indicates the number of porphyrin subunits, and $(\mathbf{b}, \mathbf{d})$ the atomic model structures of an individual hexamer chain in different viewing directions: top and side-views. Different atoms are shaded in different colours 
for the visualization (Pt: green, N: blue, Si: yellow, C: grey, and $\mathrm{H}$ atoms are omitted). The ideal Pt-Pt distance of a butadiyne-linked porphyrin dimer is $\sim 1.35 \mathrm{~nm}$, and the end-to-end Pt-Pt distance is $\sim 6.75 \mathrm{~nm}$. (c) SEM image showing several aperture positions on a holey SiN TEM grid. (e) 3D-schematic illustration showing deposited porphyrin hexamers on to a surface carbon/graphene substrate.

ADF-STEM images of the Pt-L6 deposited on to the graphene surface are shown in Figure 2a,b (see also Figure S1 for more examples). Atomic resolution image of graphene is shown in figure $2 \mathrm{c}$, from the green boxed area in $2 \mathrm{~b}$. Where the graphene surface is covered with Pt-L6 hexamers, Pt metal centres are clearly visible as higher contrast dots, as shown in Figure 2a,b. In general, Pt-L6 hexamers were well dispersed on the graphene surface, mostly with monolayer density and pretty uniform intramolecular distance, except a few highly dense or dilute regions as a typical consequence expected from a drop-cast solution method. Much of the graphene surface is covered by surface carbon residue (Figure 2d) which is observed as the lighter contrast area (outlined by yellow box in Figure 2b). Randomly distributed particles with less bright contrast than Pt atoms are also observed on surface carbon area, which are possibly C, N, and Si atoms coming of the original hexamer structure and CVD graphene contamination. However, Pt contrast is easily detected above these elements. The molecules appear to be either on the surface carbon or are surrounded by some carbon residue. Isolated Pt-L6 hexamers were selected at a moderately lowdensity area, where we could exclude the possibilities of overlapping or crossing-over among several hexamer chains, and several representative hexamers are indicated, yellow line in Figure $2 \mathrm{e}-\mathrm{i}$. 


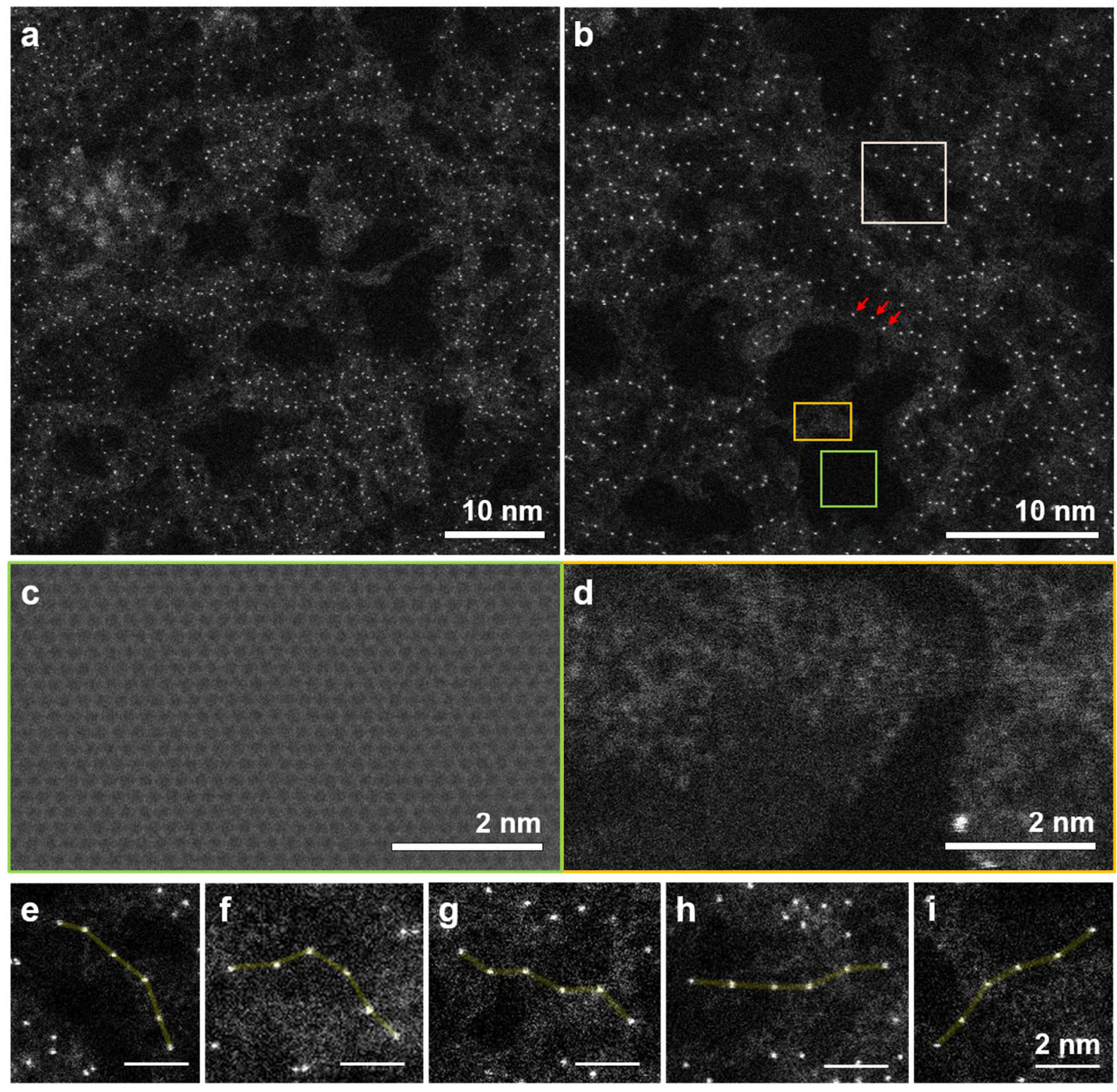

Figure 2. ADF-STEM images of Pt-porphyrin hexamers dispersed on graphene surface recorded at $80 \mathrm{kV}$. (a-b) ADF-STEM image of suspended graphene with hexamers deposited on top, taken at the same location at (a) lower and (b) higher magnifications. The darkest area (outlined by green box) in the image (b) corresponds to (c) the pristine graphene, which is covered by (d) surface amorphous carbon, which is observed as the lighter contrast area (outlined by yellow box). Pt atoms of each porphyrin unit is shown as 
bright circles (pointed by red arrows) in the image (b). (e-i) ADF-STEM images of individual Pt-porphyrin hexamers. The scale bars are $2 \mathrm{~nm}$.

To explore the behavior of Pt-L6 hexamers dispersed on graphene surface, we investigated dozens of ADF-STEM images of individual molecules at different locations on a TEM grid. We chose reasonably low concentration areas to avoid overlap of hexamers. We excluded fragmented porphyrin oligomers (i.e., $n=2-5$, shown in Figure S2) from our analysis. We also tested the electron-beam irradiation effect on a hexamer introduced by typical ADF-STEM imaging conditions to understand the degree of Pt diffusion, Figure S3. During the entire time span of about $\sim 3$ min, only small changes in both Pt-Pt interdistance and bending angles occur, which demonstrates imaging represents the intrinsic molecular structure. ADF-STEM image simulations were done using multislice methods with added noise to match the experimental. In order to consider the impact of surface carbon residue, we put 1 3 layers of amorphous carbon on monolayer graphene for the simulations in Figures $3 \mathrm{a}$ and $3 \mathrm{~b}$. Figure $3 \mathrm{c}$ shows an experimental ADF-STEM image of a single hexamer, where each Pt atom location is imaged as a bright contrast dot. Figure $3 \mathrm{~d}$ is a multislice simulated image of the atomic structure in Figure 3a, which shows that heavy Pt metal centres are predominantly discernible with the brightest contrast in STEM image under our experimental condition. Figure 3e shows experimental and simulated line profiles along the single hexamer chains in Figs $3 \mathrm{c}$ and $3 \mathrm{~d}$, with distances of $1.22,1.28 \mathrm{~nm}$ in the experiment, which was shorter than simulated value of $\sim 1.35 \mathrm{~nm}$. A histogram showing the experimental Pt-Pt inter-distance distribution within a single hexamer by investigating dozens of Pt-L6 samples is shown in Figure 3f, where the measured average Pt-Pt distance is $1.26 \pm 0.21 \mathrm{~nm}$. 

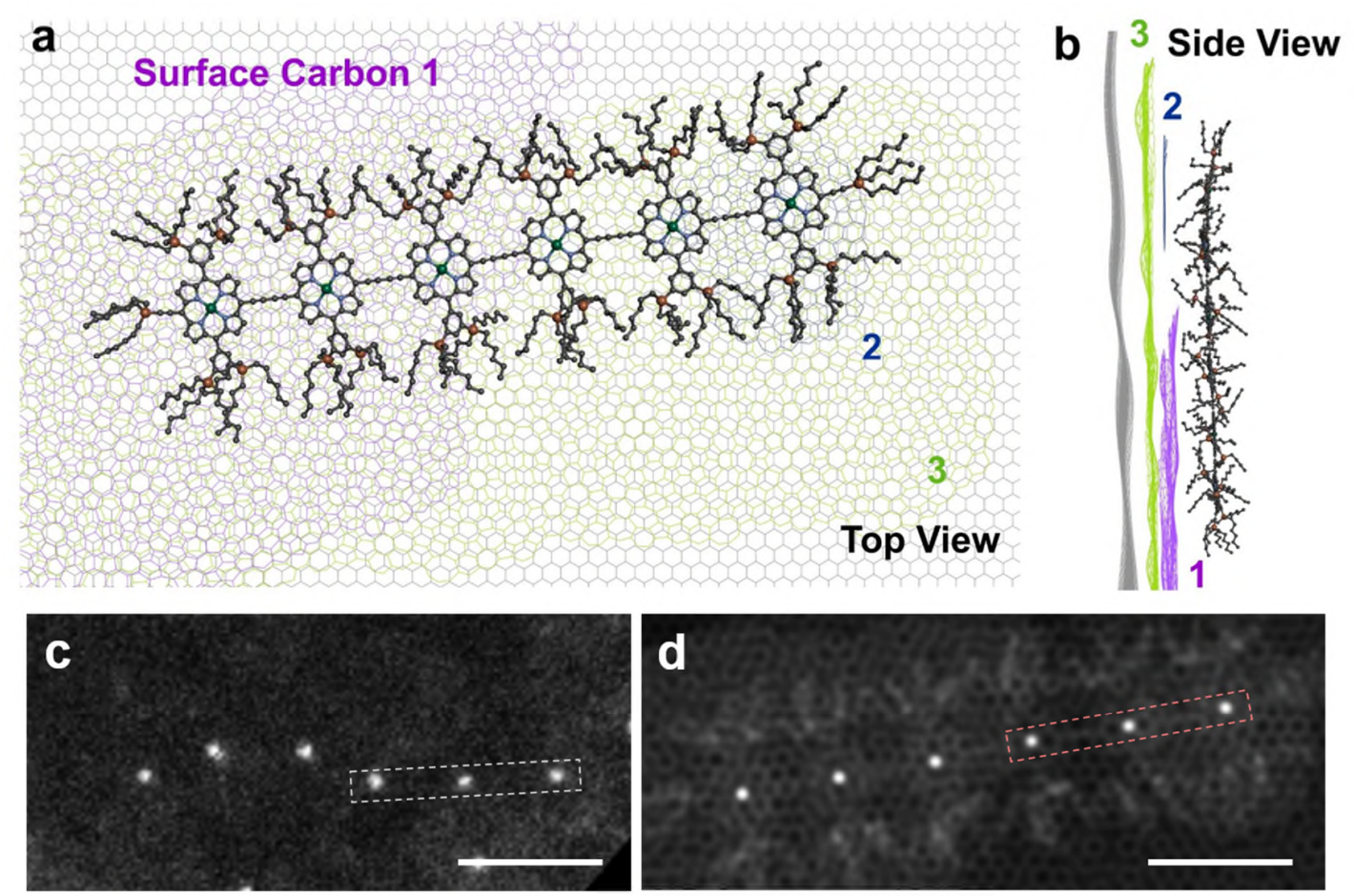

Top View
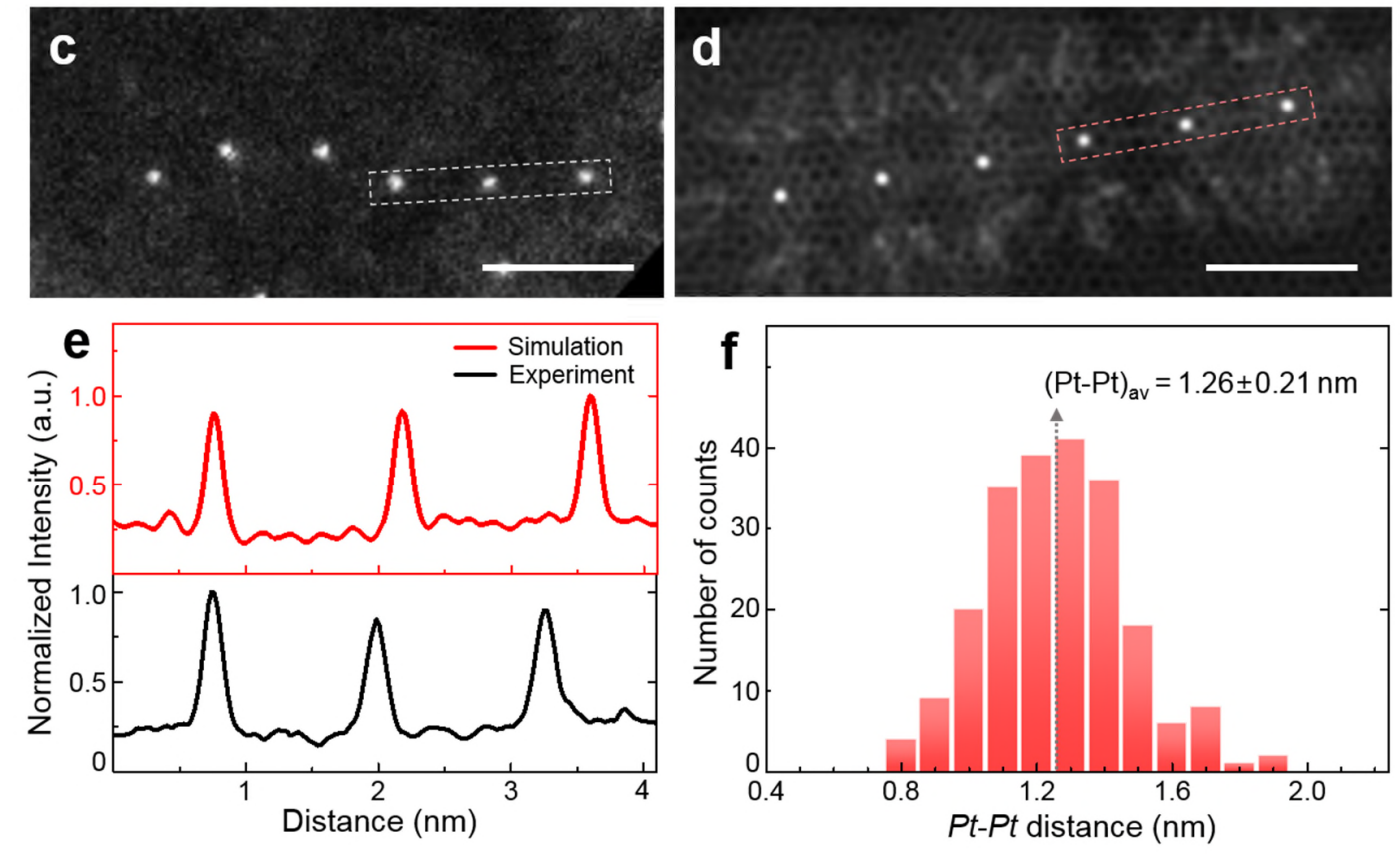

Figure 3. Individual Pt-porphyrin hexamers. (a) Top-view and (b) side-view of an atomic model used for the image simulation: Pt-L6 sitting on top of 1 3 layers of amorphous carbon on a monolayer graphene. (c) A high-resolution ADF-STEM image showing each Pt atom location in an individual hexamer with clearly resolved high contrast. (d) HR-STEM multislice image simulation performed on the structure in (a). The scale bars are $2 \mathrm{~nm}$. (e) Intensity variations obtained from the boxed region of (c) and in (d) are line profiled in black and red colour, respectively. The measured neighbouring Pt-Pt distances are 1.22, 1.28 $\mathrm{nm}$ in (c), and $\sim 1.35 \mathrm{~nm}$ in (d). (f) A histogram showing the experimental inter-distance distribution between adjacent Pt metal centres, measured from dozens of Pt-L6 samples. 
The porphyrin hexamers are not randomly distributed, but have an epitaxial relationship with the underlying graphene. Figure 4a shows a low-magnification ADF-STEM image of Pt-L6 chains aligning with each other, and the inset is the power spectrum calculated by the fast Fourier transform (FFT) obtained from this area. Figure $4 \mathrm{~b}$ is a high-resolution image taken at the region outlined by green box in Figure 4a, showing the underlying graphene lattice with its FFT pattern also presented (Note that the central beam is intentionally blocked by a black circle, in order to emphasize the diffraction spots coming from a sample itself). As highlighted with white dotted circles in each FFT pattern, hexagonal-symmetric diffraction patterns exist in addition to those of underlying graphene (see the inset FFT of Figure 4a), and each diffraction spot is well-aligned with the corresponding diffraction beams of graphene (see the inset FFT of Figure 4b) in the same angular orientation. Also, there is a diffuse circular ring (violet colour) in the inset of Figure 4a. In order to understand any reciprocal space correlations, we took a line profile along the green box in the FFT pattern, which includes the scatterings of both graphene and the other constituents. The obtained line profiles in Figure $4 \mathrm{~g}$ demonstrate that there exist three peaks. Considering scanning distortion during STEM image recording, we investigated all of the six diffraction peaks and obtained the averaged value. The measured distances correspond (1) Inter-molecular distance of $2.52 \pm 0.08 \mathrm{~nm}$, (2) Intra-molecular Pt-Pt distance of $1.13 \pm 0.01 \mathrm{~nm}$, and (3) Graphene lattice spacings of $0.21 \pm 0.00 \mathrm{~nm}$ in a real space, respectively.

Figures 4d-e are the cropped ADF-STEM images showing the alignment of hexamers along a specific crystallographic orientation. The measured intra-molecular spacing between adjacent hexamers is $2.3 \sim 2.5 \mathrm{~nm}$ roughly, which matches with the peak 1 distance within an error range. The inter-molecular diffraction spot is slightly blurred rather than forming a very sharp spot as in the case of graphene. Each scattering has broadened angular distribution, investigated in 
Figure S4. The measured full-width half-maximum (FWHM) is $35.1 \pm 16.1^{\circ}$, therefore hexamers are aligned along a specific orientation with an angular dispersion range of $35^{\circ}$. The measured peak 2 distance $(1.13 \mathrm{~nm})$ from the FFT pattern is similar to the average Pt-Pt distance of $\sim 1.26$ $\mathrm{nm}$ measured from experimental images. Finally, peak 3 distance $(0.21 \mathrm{~nm})$ indicates a lattice parameter of pristine graphene. Figure S5 schematically illustrate how the Pt-L6 molecules align along the three directions and its relationship to the FFT pattern.
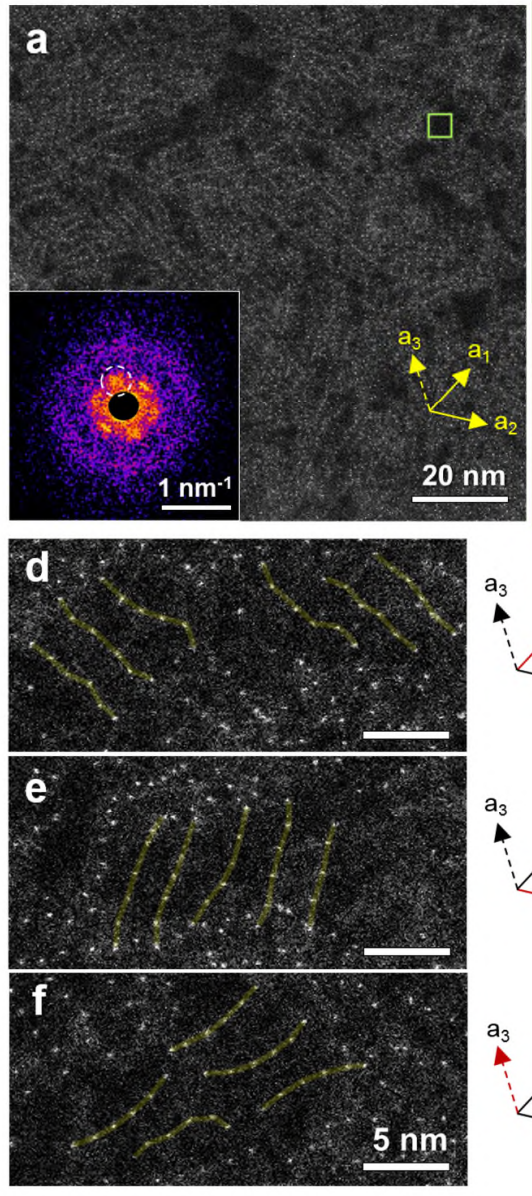


Figure 4. Remote epitaxial relationship with underlying graphene. (a) ADF-STEM image showing porphyrin hexamers aligned each other (inset: FFT pattern zooming in the reciprocal space information of longer spacing region in the real space). (b) HR-STEM image showing the graphene lattice, obtained from 
the green boxed region in (a). Inset shows the FFT pattern of graphene. Crystallographic lattice vectors are included as $\mathbf{a}_{1}$ and $\mathbf{a}_{2}$. (c) FFT pattern showing the diffraction patterns of both the underlying graphene (indicated by 3) and deposited Pt-porphyrin hexamers (indicated by 1 and 2). (d-f) The cropped ADFSTEM images showing the alignment of the hexamers along a specific crystallographic direction, which is highlighted as red coloured arrows in each panel. (g) Line profile obtained along the green boxed region in (c). The measured distances from the centre peak to each peak are 1: $0.40 \pm 0.01 \mathrm{~nm}^{-1}, 2: 0.89 \pm 0.01 \mathrm{~nm}^{-1}$, and 3: $4.69 \pm 0.02 \mathrm{~nm}^{-1}$, which correspond to $2.52 \pm 0.08 \mathrm{~nm}, 1.13 \pm 0.01 \mathrm{~nm}$, and $0.21 \pm 0.01 \mathrm{~nm}$, respectively. The indicated 1, 2, and 3 peaks represent the diffraction peaks from local alignment of hexamers, neighbouring Pt-Pt atom distance in a single hexamer, and graphene, respectively.

In order to understand the experimental contraction of inter-Pt distances measured, we present schematic atomic models in figure 5. For as-is Pt linear hexamer molecule on graphene, with the Pt-Pt distance of $\sim 1.35 \mathrm{~nm}$, the $\pi-\pi$ interactions between the graphene and porphyrin lose commensuration. However, by reducing the Pt-Pt distance to $\sim 1.23 \mathrm{~nm}$, the $\pi-\pi$ interactions between the graphene and porphyrin retain perfect commensuration. This is close to the experimental measurement of $1.25 \mathrm{~nm}$. This reduction in $\mathrm{Pt}-\mathrm{Pt}$ distance can be easily accommodated by out-of-plane bending of the interlinking carbon chains between porphyrins. 

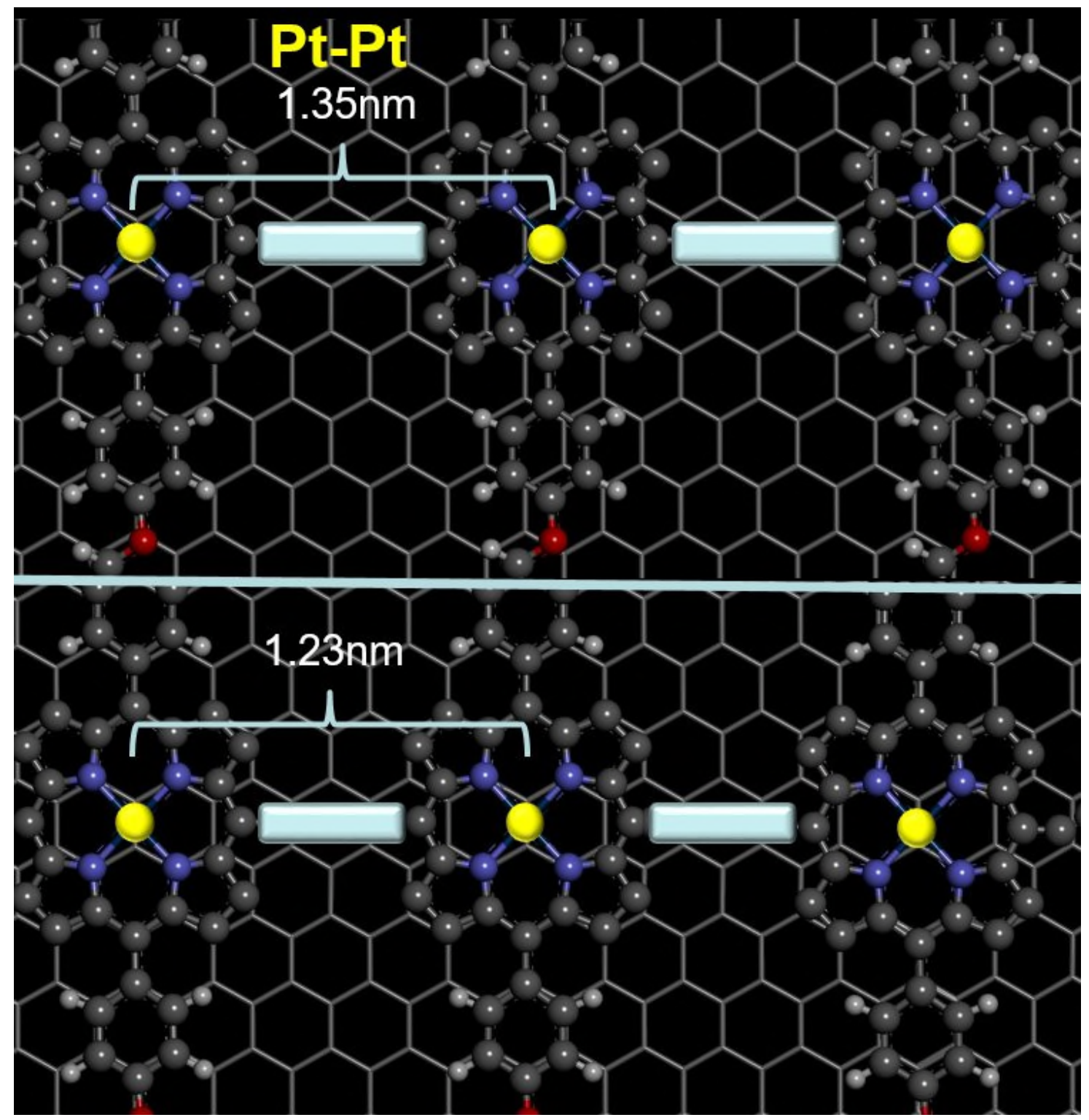

Figure 5. Schematic atomic model showing (a) unmodified Pt hexamer molecule with $1.35 \mathrm{~nm}$ separation of porphyrin centers aligned along the zig-zag direction showing poor epitaxial commensuration with graphene due to lattice mismatch. (b) Pt hexamer molecule aligned along the zig-zag direction showing perfect commensuration with graphene by shortening of the distance between the porphyrin centers to $1.23 \mathrm{~nm}$, which closely matches the experimental observation of Pt-Pt distances measured by ADF-STEM.

Figure 6a-i shows the atomic models when Pt atoms in each chain are aligned 1) side-byside, 2) offset with each other, and 3) relatively random. When two chains are brought close to each other, long side functional groups rotate to fit into a limited space or repel each other if they 
are too close. Enlarged views of the boxed regions in atomic models in Figures 6j-1 illustrate how the functional groups may interact at each vertical alignment configuration. In addition, we also considered vertical stacking of two hexamers via $\pi$ - $\pi$ interactions, Figures $6 \mathrm{~g}$ and $6 \mathrm{i}$. In this case, bulky side groups may repel each other and Pt atoms in each chain should be observed very closely located, which was not found in our study. In Figures $6 \mathrm{~m}$ and $6 \mathrm{n}$, neighbouring hexamers are mostly aligned in offset position, but not perfectly aligned, with the most probably geometry described in Figure 6e. In terms of the long-range ordering, the Pt-L6 molecules typically formed small crystalline domains, where each domain was aligned to the graphene lattice direction, Figure $60, p$. The size of these domains was between $20-100 \mathrm{~nm}$ in width, forming a polycrystalline $2 \mathrm{D}$ molecular monolayer film on graphene. This domain size is less than the grain size of graphene used and indicates it is not from graphene grain changes, but rather from the drop-casting from solution approach. 
a

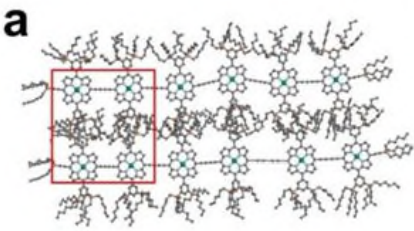

C 竞)

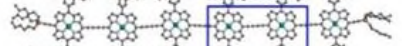
m.

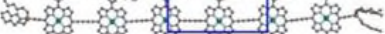
mon

e



g

Top View

3)

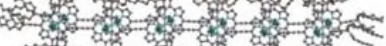
ming i

Side View

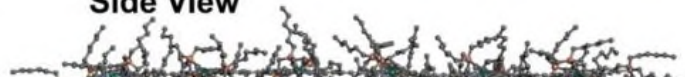
$=$ tow Dow

\section{o}
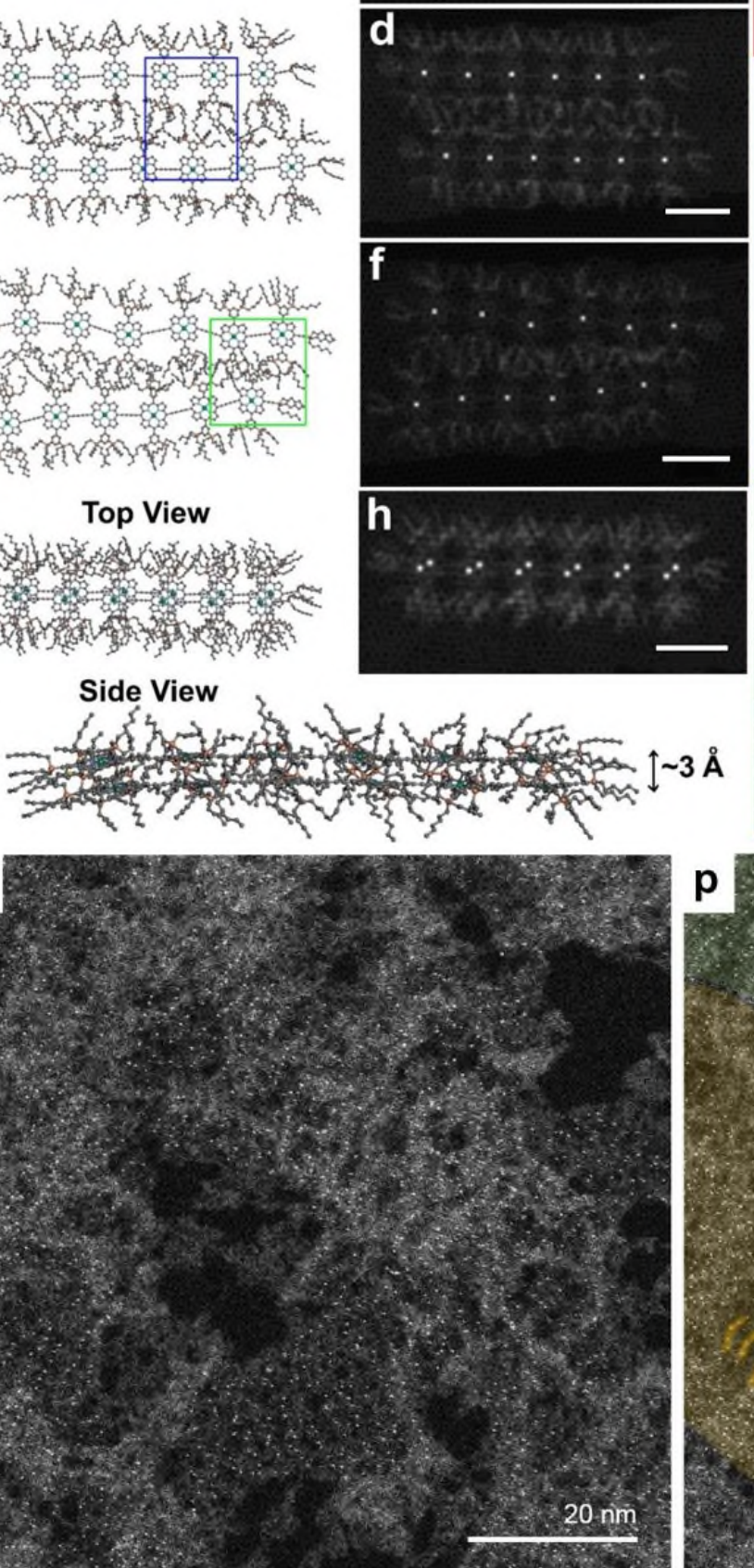


$5 \mathrm{~nm}$

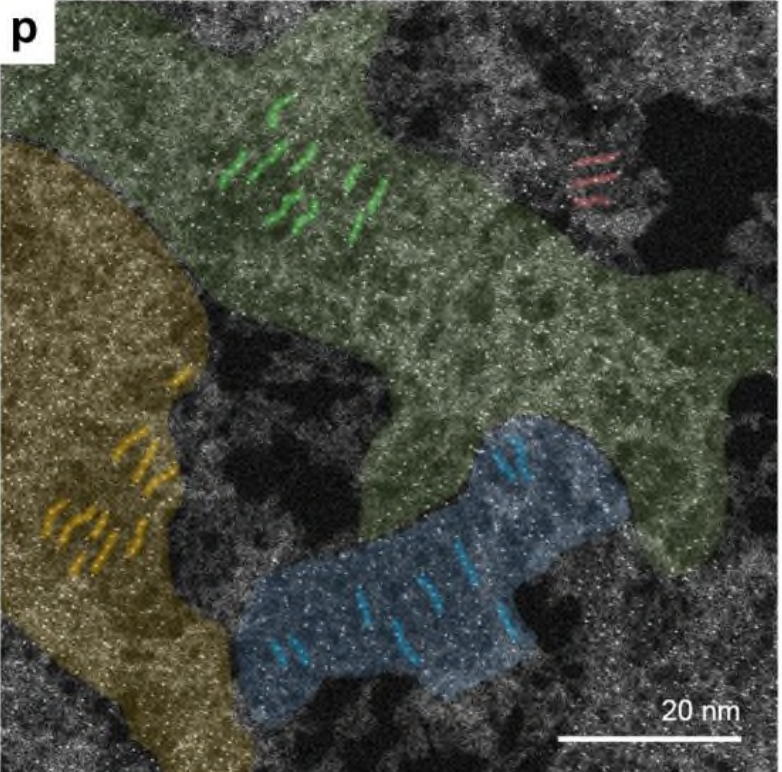

Figure 6. Ordering and alignment between the adjacent hexamers. Schematic models describing various types of vertical alignments and corresponding multislice simulated images, when Pt atoms in each chain are aligned (a-b) side-by-side, (c-d) offset each other, and (e-f) relatively random. (g-i) The atomic 
models and simulated image when the second hexamer is stacked on top of the first one. The scale bars are $2 \mathrm{~nm}$. (j-I) Enlarged views of the boxed regions in atomic models in (a), (c), and (e), showing the chainchain interactions. (m-n) ADF-STEM images showing the regions of well-aligned hexamers along the vertical direction. (o) Overview of the observation region taken at low magnification in ADF-STEM mode. (p) The same image was overlapped with coloured regions. Each colour roughly guides the boundary of the area where porphyrin hexamers are regularly aligned each other in a specific orientation. The length scales of each coloured region shown in the image are $\sim 50 \mathrm{~nm}, \sim 36 \mathrm{~nm}$, and $\sim 70 \mathrm{~nm}$ for yellow, blue, and green area respectively.

The amount of shortening of the Pt-Pt distance is $<9 \%$, which is well within the elastic limit for bonds in molecular systems at this length scale. Linear hexamer porphyrins are known to bend, with a specific persistence length that depends on their length. ${ }^{19}$ The interconnecting chains between each porphyrin are able to accommodate bending, both in the xy plane (graphene plane) and also in $\mathrm{z}$ plane (perpendicular to graphene plane). It is reasonable that the small bending of the interconnecting chains enables exact commensuration of the porphyrin rings with the underlying graphene lattice. The large molecules do not exist as rigid structures, but rather flexible wires. Furthermore, electron beam irradiation causes random displacement of Pt from the porphyrin host and this results in both contraction and enlargement of the Pt-Pt distance, not just an overall uniform contraction. The amount of Pt atom displacement is small enough that it doesn't significantly impact the overall findings of the paper. We use data from the first image of the area, minimizing the damage. We use low dose conditions (i.e low magnification and short dwell times) to minimize the beam damage. Figure $\mathrm{S} 3$ shows a time series of images revealing the small scale molecular distortion from electron beam irradiation.

\section{Conclusion}

In summary, we show that individual heavy metal atoms can be used to track complex molecules on graphene surfaces using ADF-STEM. The high contrast from the Pt atom enables 
low dose conditions to be used to minimize damage and study the epitaxial alignment of the porphyrins to the graphene. The results indicate that this approach can be expanded to a wide range of molecular systems that were previously challenging to image. The observation of epitaxy between the molecules and graphene also demonstrates strong interactions, even though the sample is deposited from solution and residual carbon surface material is likely to be present. The use of ADF-STEM to detect single molecule packing provides a complimentary approach to scanning probe microscopy, but with potential for higher through-put and atomic resolution elemental mapping.

\section{Methods}

\section{TEM Sample Preparation}

Graphene was transferred onto a holey $\mathrm{Si}_{3} \mathrm{~N}_{4}$ TEM grid (Norcada) using a PMMA support and cleaned in a high-vacuum furnace by $\mathrm{Ar} / \mathrm{H}_{2}$ flow condition at a temperature of $200^{\circ} \mathrm{C}$. A single drop of a $6 \times 10^{-7} \mathrm{M}$ concentration porphyrin solution was deposited on to the graphene TEM grid using a pipette and left to dry for $30 \mathrm{~min}$. The sample was baked in high-vacuum at $180{ }^{\circ} \mathrm{C}$ overnight to remove all the solvents.

\section{Scanning Transmission Electron Microscopy and Image Processing}

ADF-STEM imaging was performed using a JEOL ARM200F at $80 \mathrm{kV}$. CL aperture of $30 \mu \mathrm{m}$ in diameter with a convergence semi-angle of $22.5 \mathrm{mrad}$ and a beam current of $35 \mathrm{pA}$ was used for the high-resolution imaging. We used 1024 x 1024 pixel imaging and dwell times of 8-32 $\mu$ s for ADF-STEM imaging. Multislice image simulations were performed using JEMS with parameters according to experimental conditions; spherical aberration $\mathrm{Cs}=-0.003 \mathrm{~mm}$; energy spread $=$ $0.6 \mathrm{eV} ;$ probe size $=100 \mathrm{pm}$, and the convergence semiangle $=22 \mathrm{mrad}$.

\section{Supporting Information}


Supporting Information is available free of charge on the ACS Publications website. Methods for Pt-L6 synthesis, Supplementary figures include extra ADF-STEM images of molecules and graphene.

\section{Acknowledgements}

JHW thanks the support from the European Research Council (Grant: 725258). We acknowledge funding from the EPSRC (EP/N017188/1), the Swiss National Science Foundation (P2BSP2_168919), the ERC (grant 320969), and the European Community's Seventh Framework Programme H2020 ETN MSCA action under grant agreement 643238 (SYNCHRONICS). The authors acknowledge use of the South of England Analytical Electron Microscope (EP/K040375/1), within the David Cockayne Centre for Electron Microscopy, Department of Materials, University of Oxford. Alongside financial support provided by the Henry Royce Institute (Grant ref EP/R010145/1).

\section{References}

(1) Barth, J. V.; Costantini, G.; Kern, K. Engineering Atomic and Molecular Nanostructures at Surfaces. Nature 2005, 437, 671-679.

(2) Jurow, M.; Schuckman, A. E.; Batteas, J. D.; Drain, C. M. Porphyrins as Molecular Electronic Components of Functional Devices. Coord Chem. Rev. 2010, 254, 2297-2310.

(3) Yella, A.; Lee, H. -W.; Tsao, H. N.; Yi, C.; Chandiran, A. K.; Nazeeruddin, M. K.; Diau, E. W. -G.; Yeh, C. -Y.; Zakeeruddin, S. M.; Grätzel, M. Porphyrin-Sensitized Solar Cells with Cobalt (II/III)-Based Redox Electrolyte Exceed 12 Percent Efficiency. Science 2011, $334,629-634$. 
(4) Xiang, D.; Wang, X.; Jia, C.; Lee, T.; Guo, X. Molecular-Scale Electronics: From Concept to Function. Chem. Rev. 2016, 116, 4318-4440.

(5) Da Silva, E. S.; Moura, N. M. M.; Neves, M. G. P. M. S.; Coutinho, A.; Prieto, M.; Silva, C. G.; Faria, J. L. Novel Hybrids of Graphitic Carbon Nitride Sensitized with Free-Base meso-tetrakis(carboxyphenyl) Porphyrins for Efficient Visible Light Photocatalytic Hydrogen Production. Appl. Catal. B 2018, 221, 56-69.

(6) La, D. D.; Bhosale, S. V.; Jones, L. A.; Bhosale, S. V. Arginine-Induced Porphyrin-based Self-Assembled Nanostructures for Photocatalytic Applications under Simulated Sunlight Irradiation. Photochem. Photobiol. Sci. 2017, 16, 151-154.

(7) Borders, B.; Adinehnia, M.; Rosenkranz, M.; van Zijll, M.; Hipps, K. W.; Mazur, U. Photoconductive Behaviour of Binary Porphyrin Crystalline Assemblies. J. Porphyrins Phthalocyanines 2017, 21, 569-580.

(8) Limburg, B.; Thomas, J. O.; Holloway, G.; Sadeghi, H.; Sangtarash, S.; Hou, I. C.-Y.; Cremers, J.; Narita, A.; Müllen, K.; Lambert, C. J.; Briggs, G. A. D.; Mol, J. A.; Anderson, H. L. Anchor Groups for Graphene-Porphyrin Single-Molecule Transistors. Adv. Funct. Mater. 2018, 28, 1803629.

(9) Yoon, D. H.; Lee, S. B.; Yoo, K. -H.; Kim, J.; Lim, J. K.; Aratani, N.; Tsuda, A.; Osuka, A.; Kim, D. Electrical Conduction Through Linear Porphyrin Arrays. J. Am. Chem. Soc. 2003, $125,11062-11064$.

(10) Peeks, M. D.; Tait, C. E.; Neuhaus, P.; Fischer, G. M.; Hoffmann, M.; Haver, R.; Cnossen, A.; Harmer, J. R.; Timmel, C. R.; Anderson, H. L. Electronic Delocalization in the Radical Cations of Porphyrin Oligomer Molecular Wires. J. Am. Chem. 2017, 139, 10461-10471. 
(11) Iyoda, M.; Yamakawa, J.; Rahman, M. J. Conjugated Macrocycles: Concepts and Applications. Angew. Chem. Int. Ed. 2011, 50, 10522-10553.

(12) Sprafke, J. K.; Kondratuk, D. V.; Wykes, M.; Thompson, A. L.; Hoffmann, M.; Drevinskas, R.; Chen, W. -H.; Yong, C. K.; Kärnbratt, J.; Bullock, J. E.; Malfois, M.; Wasielewski, M. R.; Albinsson, B.; Herz, L. M.; Zigmantas, D.; Beljonne, D.; Anderson, H. L. Belt-Shaped $\pi$-Systems: Relating Geometry to Electronic Structure in a SixPorphyrin Nanoring. J. Am. Chem. Soc. 2011, 133, 17262-17273.

(13) Bols, P. S.; Anderson, H. L. Shadow Mask Templates for Site Selective Metal Exchange in Magnesium Porphyrin Nanorings. Angew. Chem. Int. Ed. 2018, 57, 7874-7877.

(14) Bols, P. S.; Anderson, H. L. Template-Directed Synthesis of Molecular Nanorings and Cages. Acc. Chem. Res. 2018, 51, 2083-2092.

(15) Gimzewski, J. K.; Joachim, C. Nanoscale Science of Single Molecules Using Local Probes. Science 1999, 283, 1683-1688.

(16) De Feyter, S.; De Schryver, F. C. Two-Dimensional Supramolecular Self-Assembly Probed by Scanning Tunneling Microscopy. Chem. Soc. Rev. 2003, 32, 139-150.

(17) De Feyter, S.; De Schryver, F. C. Self-Assembly at the Liquid/Solid Interfaces: STM Reveals. J. Phys. Chem. B. 2005, 109, 4290-4302.

(18) Spillmann, H.; Kiebele, A.; Stöhr, M.; Jung, T. A.; Bonifazi, D.; Cheng, F.; Diederich, F. A Two-Dimensional Porphyrin-Based Porous Network Featuring Communicating Cavities for the Templated Complexation of Fullerenes. Adv. Mater. 2006, 18, 275-279.

(19) Saywell, A.; Sprafke, J. K.; Esdaile, L. J.; Britton, A. J.; Rienzo, A.; Anderson, H. L.; O’Shea, J. N.; Beton, P. H. Conformation and Packing of Porphyrin Polymer Chains 
Deposited Using Electrospray on a Gold Surface. Angew. Chem. Int. Ed. 2010, 49, 91369139.

(20) Huang, H.; Wong, S. L.; Chen, W.; Wee, A. T. S. LT-STM Studies on SubstrateDependent Self-Assembly of Small Organic Molecules. J. Phys. D: Appl. Phys. 2011, 44, 464005.

(21) Xu, L.; Yang, L.; Lei, S. Self-Assembly of Conjugated Oligomers and Polymers at the Interface: Structure and Properties. Nanoscale 2012, 4, 4399.

(22) Garah, M. El.; Santana Bonilla, A.; Ciesielski, A.; Gualandi, A.; Mengozzi, L.; Fiorani, A.; Lurlo, M.; Marcaccio, M.; Gutierrez, R.; Rapino, S.; Calvaresi, M.; Zerbetto, F.; Cuniberti, G.; Cozzi, P. G.; Paolucci, F.; Samori, P. Molecular Design Driving Tetraporphyrin Self-assembly on Graphite: a Joint STM, Electrochemical and Computational Study. Nanoscale 2016, 8, 13678.

(23) Khlobystov, A. N.; Britz, D. A.; Briggs, G. A. D. Molecules in Carbon Nanotubes. Acc. Chem. Res. 2005, 38, 901-909.

(24) Warner, J. H.; Ito, Y.; Zaka, M.; Ge, L.; Akachi, T.; Okimoto, H.; Porfyrakis, K.; Watt, A. A. R. Shinohara, H.; Briggs, G. A. D. Rotating Fullerene Chains in Carbon Nanopeapods. Nano Lett. 2008, 8, 2328-2335.

(25) Warner, J. H.; Ito, Y.; Rümmeli, M. H.; Gemming, T.; Büchner, B.; Shinohara, H.; Briggs, G. A. D. One-Dimensional Confined Motion of Single Metal Atoms Inside DoubleWalled Carbon Nanotubes. Phys. Rev. Lett. 2009, 102, 195504.

(26) Allen, C. S.; Ito Y.; Robertson, A. W.; Shinohara, H.; Warner, J. H. Two-Dimensional Coalescence Dynamics of Encapsulated Metallofullerenes in Carbon Nanotubes. ACS Nano 2011, 5, 10084-10089. 
(27) Umeyama, T.; Baek, J.; Yuta, S.; Suenaga, K.; Abou-Chahine, F.; Tkachenko, N. V.; Lemmetyinen, H.; Imahori, H. Molecular Interactions on Single-walled Carbon Nanotubes Revealed by High-Resolution Transmission Microscopy. Nat. Commun. 2015, $6,7732$.

(28) Nair, R. R.; Blake, P.; Blake, J. R.; Zan, R.; Anissimova, S.; Bangert, U.; Golovanov, A. P.; Morozov, S. V.; Geim, A. K.; Novoselov, K. S.; Latychevskaia, T. Graphene as a Transparent Conductive Support for Studying Biological Molecules by Transmission Electron Microscopy. Appl. Phys. Lett. 2010, 97, 153102.

(29) Turchanin, A.; Käfer, D.; El-Desawy, M.; Wöll, C.; Witte, G.; Gölzhäuser, A. Molecular Mechanisms of Electron-Induced Cross-Linking in Aromatic SAMs. Langmuir 2009, 25, $7342-7352$.

(30) Skowron, S. T.; Chamberlain, T. W.; Biskupek, J.; Kaiser, U.; Besley, E.; Khlobystov, A. N. Chemical Reactions of Molecules Promoted and Simultaneously Imaged by the Electron Beam in Transmission Electron Microscopy. Acc. Chem. Res. 2017, 50, $1797-$ 1807.

(31) Wang, H.; Li, L.; Guan, J.; Jiang, H.; Shen, R.; Ding, X.; Li, J.; Li, Y. Investigation on Molecular Structures of Electron-Beam-Irradiated Low-Density Polyethylene by Rheology Measurements. Ind. Eng. Chem. Res. 2018, 57, 4298-4310.

(32) Matei, D. G.; Weber, N. -E.; Kurasch, S.; Wundrack, S.; Woszczyna, M.; Grothe, M.; Weimann, T.; Ahlers, F.; Stosch, R.; Kaiser, U.; Turchanin, A. Functional Single-Layer Graphene Sheets from Aromatic Monolayers. Adv. Mater. 2013, 25, 4146-4151.

(33) Lackinger, M. On-surface Polymerization - A Versatile Synthetic Route to TwoDimensional Polymers. Polym. Int. 2015, 64, 1073-1078. 
(34) Hernández, E.; Meunier, V.; Smith, B. W.; Rurali, R.; Terrones, H.; Buongiorno Nardelli, M.; Terrones, M.; Luzzi, D. E.; Charlier, J. -C. Fullerene Coalescence in Nanopeapods: A Path to Novel Tubular Carbon. Nano Lett. 2003, 3, 1037-1042.

(35) Terrones, M. Visualizing Fullerene Chemistry. Nat. Chem. 2010, 2, 82.

(36) Koshino, M.; Niimi, Y.; Nakamura, E.; Kataura, H.; Okazaki, T.; Suenaga, K.; Iijima, S. Analysis of the Reactivity and Selectivity of Fullerene Dimerization Reactions at the Atomic Level. Nat. Chem. 2010, 2, 117-124.

(37) Warner, J. H.; Plant, S. R.; Young, N. P.; Porfyrakis, K.; Kirkland, A. I.; Briggs, G. A. D. Atomic Scale Growth Dynamics of Nanocrystals Within Carbon Nanotubes. ACS Nano 2011, 5, 1410-1417.

(38) Ryll, H.; Simson, M.; Hartmann, R.; Holl, P.; Huth, M.; Ihle, S.; Kondo, Y.; Kotula, P.; Liebel, A.; Müller-Caspary, K.; Rosenauer, A.; Sagawa, R.; Schmidt, J.; Soltau, H.; Strüder, L. A pnCCD-based, Fast Direct Single Electron Imaging Camera for TEM and STEM. J. Instrum. 2016, 11, P04006.

(39) Nellist, P. D.; Pennycook, S. J. The Principles and Interpretation of Annular Dark-field Z-Contrast Imaging. Adv. Imaging Electron Phys. 2000, 113, 147-203.

(40) Gerkman, M. A.; Sinha, S.; Warner, J. H.; Han, G. G. D. Direct Imaging of Photoswitching Molecular Conformations Using Individual Metal Atom Markers. ACS Nano 2019, 13, 87-96.

(41) Mirzayev, R.; Mustonen, K.; Monazam, M. R. A.; Mittelberger, A.; Pennycook, T. J.; Mangler, C.; Susi, T.; Kotakoski, J.; Meyer, J. C. Buckyball Sandwiches. Sci. Adv. 2017, 3, e1700176. 
(42) Sinha, S.; Sheng, Y.; Griffiths, I.; Young, N. P.; Zhou, S.; Kirkland, A. I.; Porfyrakis, K.; Warner, J. H. In situ Atomic-level Studies of Gd atom Release and Migration on Graphene from a Metallofullerene Precursor. ACS Nano 2018, 12, 10439-10451.

(43) Furuya, F. R.; Miller, L. L.; Hainfeld, J. F.; Christopfel, W. C.; Kenny, P. W.; Use of Ir4(CO)11 to Measure the Lengths of Organic Molecules with a Scanning Transmission Electron Microscope, JACS, 1988, 110, 641-643

(44) Anderson, H. L. Building Molecular Wires From the Colours of Life: Conjugated Porphyrin Oligomers. Chem. Commun. 1999, 2323-2330.

(45) Taylor, P. N.; Huuskonen, J.; Rumbles, G.; Aplin, R. T.; Williams, E.; Anderson, H. L. Conjugated Porphyrin Oligomers From Monomer to Hexamer. Chem. Commun. 1998, 909-910.

\section{TOC graphic}



